potential equation and corresponds to the supersonic flow past a cone in the range of Mach numbers for which the shock wave at the nose is attached to the cone. The only lost solutions of the axially symmetric potential flow equation are thus seen to be a rectilinear flow along the axis, a.two dimensional line source on the axis, and the Taylor Maccoll flow past a cone. The first two flows may be superimposed, but the result is of little interest. Only the third conical flow is an essentially three dimensional flow pattern.

In conclusion it can be stated that all the lost solutions of axially symmetric irrotational compressible flow are already well known solutions. This result was apparently known to Bateman; however, it does not appear in any of his writings. A discussion of another class of three dimensional lost solutions, conical flows, has been given by Busemann. ${ }^{4}$

\title{
A GENERAL STABILITY CRITERION FOR LINEAR OSCILLATING SYSTEMS WITH CONSTANT TIME LAG*
}

\section{By H. I. ANSOFF AND J. A. KRUMHANSL (Brown University)}

Several formulations ${ }^{1}$ have recently been given for the stability criterion of a lumpedparameter linear oscillating system with constant time lag given by the equation

$$
I y^{\prime \prime}(t)+R y^{\prime}(t)+K y(t)=-S y^{\prime}(t-\tau),
$$

where $I, R, K, S$ and $\tau$ are real positive constants and $S y^{\prime}(t-\tau)$ is the feedback term.

By applying Cauchy's index theorem to the operational form of (1) it is possible to generalize the discussion to feedback proportional to any derivative of the dependent variable taken at time $t-\tau$; this will be called a retarded derivative. It is found that the resulting stability criterion can be written in an easily computed form and that the permissible range in the time lag $\tau$ can be stated explicitly.

Equation (1) is generalized to

$$
I y^{\prime \prime}(t)+R y^{\prime}(t)+K y(t)=-S y^{(n)}(t-\tau),
$$

where $n$ is allowed any integer positive value. Letting $F(p)$ be the Laplace transform of $y(t)$, one obtains ${ }^{2}$

$$
F(p)=\frac{L(p)}{Y(p)+S p^{n} e^{-p \tau}}=\frac{L(p)}{Y(p)} \frac{1}{1+S p^{n} e^{-p \tau} / Y(p)},
$$

where

$$
\begin{gathered}
L(p)=I p y(0)+I y^{\prime}(0)+R y(0) \\
Y(p)=I p^{2}+R p+K
\end{gathered}
$$

${ }^{4}$ A. Busemann, Aerodynamischer Auftrieb bei Überschallgeschwindigkeit, Luftfahrtforschung, 12, 210 (1935).

${ }^{*}$ Received Feb. 27, 1948

${ }^{1}$ See references [1] and [2]. In the following, numbers in square brackets refer to the Bibliography at the end of this paper.

${ }^{2}$ The Laplace transform is defined here as in [3]. For other examples of this procedure see reference [4]. 
By Mellin's inversion theorem

$$
y(t)=\frac{1}{2 \pi i} \int_{\Gamma} \frac{L(p)}{Y(p)} \frac{e^{p t} d p}{1+S p^{n} e^{-p \tau} / Y(p)} \quad \text { for } \quad t>0,
$$

where $\Gamma$ is a contour enclosing all of the poles of the integrand in the $p$-plane.

Evaluation of this integral by the method of residues yields an expression of the form

$$
y(t)=\sum_{0}^{n} A_{n} \exp \left(p_{n} t\right),
$$

where $p_{n}$ are the poles of the integrand of (5). It is easily shown [5] that in general there is an infinity of these poles. Among these poles, however, only $p_{n}$ with positive real parts will cause (6) to be unstable. The subsequent investigation is therefore devoted to a formulation of conditions under which the system has no $p_{n}$ with positive real parts.

The functions $L(p)$ and $e^{p t}$ in (5) have no poles in the finite $p$ plane, and $Y(p)$ has no roots in the right half of the $p$ plane, as shown by (4). Thus a necessary and sufficient condition for the stability of $(6)$ is that

$$
1+S \frac{p^{n} e^{-p r}}{Y(p)}=0
$$

have no roots in the right half of the $p$ plane.

In order to determine the roots of (7), we introduce the Cauchy index theorem [6] which may be simplified as follows:

Hypothesis. 1) $w=f(p)$ is an analytic function in a simply connected domain $D$ bounded by a contour $\beta$, and

2) $f(p) \neq 0$ for $p$ on $\beta$.

Conclusion. If $p$ traverses $\beta$ in a counter-clockwise direction, then $w$ will traverse a closed curve in the $w$-plane. Further, the number of zeros of $f(p)$ in $D$ is equal to the net number of times the contour in the w-plane encircles the origin.

For $\beta$ we choose a Bromwich contour in the $p$ plane consisting of the imaginary axis $^{3}$ and an infinite semi-circle in the right half of the plane with center at the origin.

Applying the theorem to (7), we note that the number of roots of (7) with positive real parts is equal to the number of times the graph of the function

$$
f(p)=\frac{S p^{n} e^{-p \tau}}{I p^{2}+R p+K}
$$

encloses the point $(-1,0)$ as $p$ traverses $\beta$. It can be shown [5] that $f(p) \rightarrow 0$ for all values of $n$, as the radius $R$ of the semi-circular part of $\beta$ tends to $\infty$. It remains to examine $f(p)$ on the imaginary axis.

If we let $p=i \alpha$, where $\alpha$ is real, Eq. (8) can be written

$$
f(i \alpha)=\frac{S \alpha^{n} e^{i \beta}}{\left[\left(K-I \alpha^{2}\right)^{2}+(R \alpha)^{2}\right]^{\frac{1}{3}}},
$$

where

${ }^{3}$ It is shown in [5] that roots on the imaginary axis occur only for a very critical combination of the parameters of the system. 


$$
\begin{gathered}
\beta=-\alpha \tau+\phi+n \pi / 2, \\
\tan \phi=\frac{R \alpha}{K-I \alpha^{2}},
\end{gathered}
$$

and $n$ corresponds to the order of the feedback term

The graph of (9) for any fixed $n$ is a sensed spiral with a variable amplitude given by $|f(i \alpha)|$. Figure 1 shows typical graphs for $n=0,1,2$, respectively, in the range

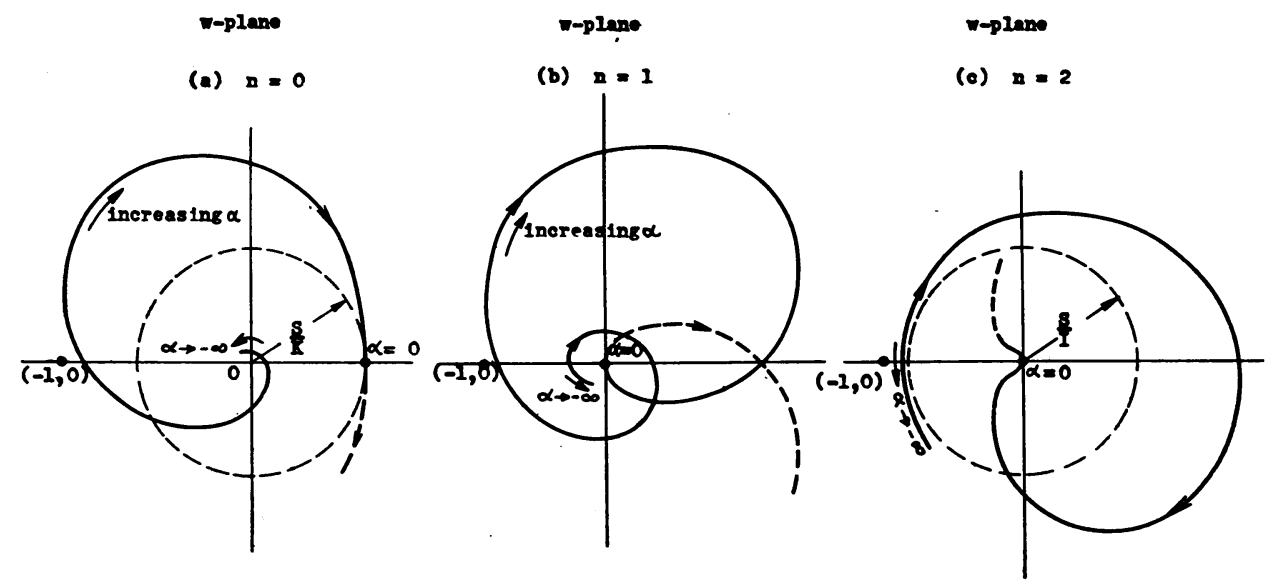

FIG. 1. $f(i \alpha)$ vs. $\alpha$.

$-\infty \leqq \alpha \leqq 0$. As $\alpha$ increases from 0 to $\infty$, each graph traces the second branch of the spiral which is symmetrical to the first branch with respect to the real axis. Figure 2 shows typical graphs of $|f(i \alpha)|$ versus $\alpha$ for $n=0,1,2$ and $n>2$.
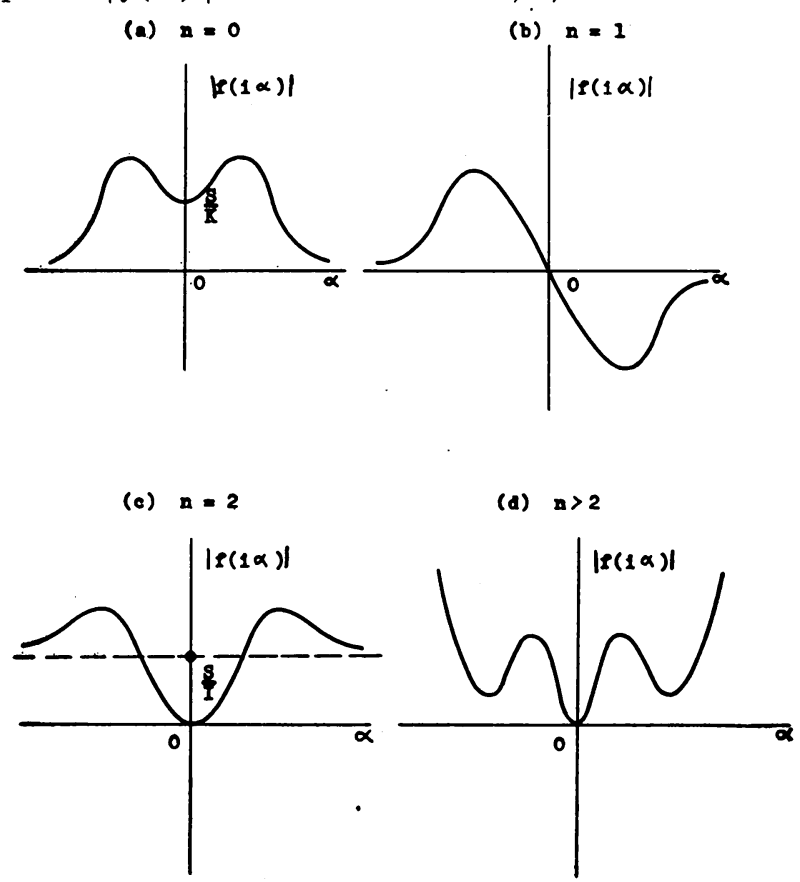

Fig. 2. $|f(i \alpha)|$ vs. $\alpha$. 
Now if $p \rightarrow \pm i \infty$, then

$$
f(p) \rightarrow \frac{S}{I} p^{n-2} \quad \text { and } \quad|f(p)| \rightarrow \infty
$$

except for $n=0,1,2$. Thus, for $n>2$, as $p$ traces the imaginary axis, the graph of Eq. (9) will always enclose the point $(-1,0)$. This means that $(7)$ will always have roots in the right half-plane. The general stability criterion for Eq. (2) can now be formulated.

If the feedback term is proportional to the retarded derivative $y^{(n)}(t-\tau)$, the system will be unconditionally unstable whenever $n>2$.

For $n=0,1,2$ separate stability criteria have to be formulated in each case. The general procedure is to set

$$
|f(i \alpha)|=1
$$

and solve for $\alpha$. In general, one obtains four real roots, two for each branch of the spiral, symmetric with respect to the real axis. It can be shown [5] that the angle $\beta$ of the spiral is monotone in $\alpha$ and that each branch of the spiral will have a sector, say $\beta_{1} \leqq \beta \leqq \beta_{2}$, within which $|f(i \alpha)| \geqq 1$. This is illustrated in Fig. 3. The stability criterion is now reduced to the condition that this sector does not enclose the negative real axis.

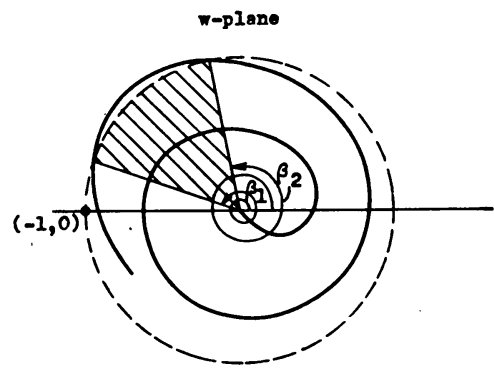

Frg. 3.

A Stable Configuration for $n=1$.

In degenerate cases Eq. (10) may have only one real root for each branch of the spiral and the criterion is accordingly simplified. If (10) has no real roots, the system is unconditionally stable.

(Because of the symmetry of each spiral with respect to the real axis, it is sufficient to formulate criteria for one branch only).

A detailed application for the above procedure to each case of feedback will be found in reference [5]. Summarized below are the allowable ranges in $\tau$ for which the system will be stable. The expressions are written for the branch of the spiral on which $\alpha>0$. We let $\alpha_{1}$ and $\alpha_{2}$ designate the two roots of (10) on this branch and $\phi_{1}$ and $\phi_{2}$ be the corresponding phase angles computed from $(9 \mathrm{c})$.

Feedback proportional to $y(t-\tau) ; n=0$.

(a) $\alpha_{1}, \alpha_{2} \neq 0$, real, $\alpha_{1}>\alpha_{2}$. In this case $S<K$ and $2 I K>R^{2}$. The range in $\tau$ is

$$
\alpha_{2}^{-1}\left[\phi_{2}+(2 n-1) \pi\right]<\tau<\alpha_{1}^{-1}\left[\phi_{1}+(2 n+1) \pi\right],
$$


where $n$ is allowed integer positive values. Examination of (11) shows that for any given system (fixed $\alpha_{1}, \alpha_{2}, \phi_{1}$ and $\phi_{2}$ ) $\tau$ is allowed a band spectrum of values for increasing $n$ with the band width given by (11).

(b) $\alpha_{1}=\alpha_{2} \neq 0$, real. Then $S \leqq K$ and the allowable values of $\tau$ are given by

$$
\tau \neq \alpha^{-1}(n \pi+\phi),
$$

where $n$ is any odd integer. In this case the "instability" sector has degenerated into a straight line.

(c) $\alpha_{1}>0$, real, $\alpha_{2}$ imaginary. Then $S>K$ and the range is

$$
0<\tau<\alpha_{1}^{-1}\left(\pi+\phi_{1}\right) \text {. }
$$

Here the sectors from the two branches have degenerated into one which is symmetrical with respect to the positive real axis.

(d) $\alpha_{1}$ and $\alpha_{2}$ both imaginary. This occurs if and only if

$$
S<K \quad \text { and } \quad R^{2}>2 I K \text {. }
$$

The system is stable for all values of $\tau$.

Feedback proportional to $y^{\prime}(t-\tau) ; n=1$.

(a) $\alpha_{1}, \alpha_{2} \neq 0$, real, $\alpha_{1}>\alpha_{2}$. In this case $R<S$. Equation (11) applies.

(b) $\alpha_{1}=\alpha_{2} \neq 0$, real. A sufficient condition for this to happen is $R=S$. Equation (12) applies.

(c) $\alpha_{1}, \alpha_{2}$ both imaginary. This occurs if and only if $R>S$. The system is stable for all values of $\tau$.

Feedback proportional to $y^{\prime \prime}(t-\tau) ; n=2$.

As seen from Fig. 1(c) and Fig. 2(c) the system is unconditionally unstable if $S>I$.

(a) $\alpha_{1}, \alpha_{2} \neq 0$, real, $\alpha_{1}>\alpha_{2}$. Then $S<I$ and $R^{2}<2 I K$. Equation (11) applies.

(b) $\alpha_{1}=\alpha_{2} \neq 0$, real. A sufficient condition is $S=I$ and $R^{2}=2 I K$. Equation (12) applies.

(c) $\alpha_{1}, \alpha_{2}$ both imaginary. This occurs if and only if

$$
S<I \quad \text { and } \quad R^{2}>2 I K
$$

The system is stable for all values of $\tau$.

\section{BIBLIOGRAPHY}

[1] Seymore Sherman, $A$ note on stability calculations and time lag, Q. Appl. Math. 5, 92 (1947).

[2] N. Minorsky, Self excited oscillations in dynamical systems, J. Appl. Mech. 64, A 65-71 (1942).

[3] M. F. Gardner and J. L. Barnes, Transients in linear systems, Wiley \& Sons, New York, 1942.

[4] L. McColl, Fundamental theory of servomechanisms, Van Nostrand Co., New York, 1945.

[5] H. I. Ansoff, Stability of linear oscillating systems with constant time lag, this is a more detailed version of the present paper which has been submitted to the Journal of Applied Mechanics.

[6] A. Hurwitz, Funktionentheorie, J. Springer, Berlin, 1929, p. 106. 